\title{
Pulmonary artery to aorta ratio for the detection of pulmonary hypertension: cardiovascular magnetic resonance and invasive hemodynamics in heart failure with preserved ejection fraction
}

Gültekin Karakus ${ }^{2+}$, Andreas A. Kammerlander ${ }^{1+}$, Stefan Aschauer ${ }^{1}$, Beatrice A. Marzluf ${ }^{3}$, Caroline Zotter-Tufaro ${ }^{1}$, Alina Bachmann ${ }^{1}$, Aleks Degirmencioglu², Franz Duca ${ }^{1}$, Jamil Babayev ${ }^{1}$, Stefan Pfaffenberger ${ }^{1}$,

Diana Bonderman ${ }^{1}$ and Julia Mascherbauer ${ }^{1 *}$

\begin{abstract}
Background: Previous work indicates that dilatation of the pulmonary artery (PA) itself or in relation to the ascending aorta (PA:Ao ratio) predicts pulmonary hypertension (PH). Whether these results also apply for heart failure with preserved ejection fraction (HFpEF) is unknown.

In the present study we evaluated the diagnostic and prognostic power of PA diameter and PA:Ao ratio on top of right ventricular (RV) size, function, and septomarginal trabeculation (SMT) thickness by cardiovascular magnetic resonance (CMR) in HFpEF.
\end{abstract}

Methods and Results: 159 consecutive HFpEF patients were prospectively enrolled. Of these, 111 underwent CMR and invasive hemodynamic evaluation.

By invasive assessment $64 \%$ of patients suffered from moderate/severe PH (mean pulmonary artery pressure (mPAP) $\geq 30 \mathrm{mmHg}$ ). Significant differences between groups with and without moderate/severe PH were observed with respect to PA diameter $(30.9 \pm 5.1 \mathrm{~mm}$ versus $26 \pm 5.1 \mathrm{~mm}, p<0.001)$, PA:Ao ratio $(0.93 \pm 0.16$ versus $0.78 \pm$ $0.14, p<0.001)$, and SMT diameter $(4.6 \pm 1.5 \mathrm{~mm}$ versus $3.8 \pm 1.2 \mathrm{~mm} ; p=0.008)$. The strongest correlation with mPAP was found for PA:Ao ratio $(r=0.421, p<0.001)$. By ROC analysis the best cut-off for the detection of moderate/severe $\mathrm{PH}$ was found for a PA:Ao ratio of 0.83 .

Patients were followed for $22.0 \pm 14.9$ months. By Kaplan Meier analysis event-free survival was significantly worse in patients with a PA:Ao ratio $\geq 0.83$ (log rank, $p=0.004$ ). By multivariable Cox-regression analysis PA:Ao ratio was independently associated with event-free survival $(p=0.003)$.

Conclusion: PA:Ao ratio is an easily measureable noninvasive indicator for the presence and severity of PH in $\mathrm{HFpEF}$, and it is related with outcome.

Keywords: Cardiovascular magnetic resonance, Pulmonary hypertension, PA:Ao ratio

\footnotetext{
* Correspondence: julia.mascherbauer@meduniwien.ac.at

${ }^{\dagger}$ Equal contributors

'Department of Cardiology, Medical University of Vienna, Waehringer Guertel

18-20, 1090 Vienna, Austria

Full list of author information is available at the end of the article
}

\section{Ciomed Central}

(c) 2015 Karakus et al. Open Access This article is distributed under the terms of the Creative Commons Attribution 4.0 International License (http://creativecommons.org/licenses/by/4.0/), which permits unrestricted use, distribution, and reproduction in any medium, provided you give appropriate credit to the original author(s) and the source, provide a link to the Creative Commons license, and indicate if changes were made. The Creative Commons Public Domain Dedication waiver (http://creativecommons.org/publicdomain/zero/1.0/) applies to the data made available in this article, unless otherwise stated. 


\section{Background}

About $50 \%$ of patients presenting with symptoms of heart failure are diagnosed with heart failure with preserved ejection fraction (HFpEF). HFpEF is characterized by impaired diastolic function due to abnormal relaxation of the left ventricle (LV) as well as increased chamber stiffness [1]. The pathophysiology underlying HFpEF is still incompletely understood. Arterial hypertension, coronary artery disease and diabetes mellitus seem to play an important role [2]. The development of postcapillary pulmonary hypertension $(\mathrm{PH})$ is a common feature of HFpEF and associated with substantial morbidity and mortality, frequent hospitalizations and an impaired quality of life [3-6].

Accurate assessment of $\mathrm{PH}$ requires invasive measurement of pulmonary artery (PA) pressure and pulmonary arterial wedge pressure (PAWP) [7]. Because of the invasive nature of this procedure the majority of HFpEF patients today are not evaluated by right heart catheter (RHC). Thus, diagnosis of HFpEF-PH mostly relies upon echocardiography. The estimation of PA pressure by echocardiography is a routine measurement, however, the accuracy of this method is limited [8] and the echocardiographic estimation of pulmonary vascular resistance is unreliable [9]. Therefore, an alternative straightforward non-invasive technique in addition to echocardiography is desirable to predict the likelihood of $\mathrm{PH}$ in $\mathrm{HFpEF}$ patients.

Cardiovascular magnetic resonance (CMR) is the current gold-standard technique to assess right heart dimensions, function, wall thickness, as well as the dimensions of the great arteries [10]. Previous work shows that dilatation of the pulmonary artery (PA) itself or in relation to the diameter of the ascending aorta (PA:Ao ratio) predicts $\mathrm{PH}$ [11-15]. Furthermore, right ventricular septomarginal trabeculation (SMT) mass has been identified as a CMR-derived marker of PH [16].

The present study was intended to determine the usefulness and prognostic significance of easily measureable variables such as PA diameter and PA:Ao ratio on top of CMR derived right ventricular (RV) dimension, ejection fraction, SMT dimensions, and RV free wall thickness (RVFW) in a prospective, well defined HFpEF cohort.

\section{Methods}

\section{Study design}

This was a prospective observational study performed at the Medical University of Vienna. All participants gave written informed consent. The institutional review board approved the study protocol (EK \#796/2010).

\section{Patients}

Consecutive patients with suspected HFpEF-PH were invited to participate. HFpEF was diagnosed in the presence of: (1) symptoms or signs of heart failure; (2) normal or mildly reduced left ventricular (LV) systolic function (LV ejection fraction (EF) $>50 \%$ ); (3) evidence of abnormal LV relaxation or diastolic stiffness $\left(\mathrm{E} / \mathrm{e}^{\prime}>8\right.$ by echocardiography; and (4) NT-proBNP serum levels $\geq 220 \mathrm{pg} / \mathrm{mL}$ [17]. Reasons for exclusion included pacemaker or other conditions precluding patients from CMR, regional wall motion abnormalities, significant coronary artery disease, significant valvular or congenital heart disease, or a glomerular filtration rate (GFR) below $30 \mathrm{~mL} / \mathrm{min} / 1.73 \mathrm{~m}^{2}$. All patients underwent ventilation-perfusion scans and lung function testing including diffusing capacity of the lung for carbon monoxide to rule out chronic thromboembolic $\mathrm{PH}$ and $\mathrm{PH}$ due to significant lung disease.

\section{Right and left heart catheterization}

PAWP, mean pulmonary artery pressure (mPAP), and cardiac output $(\mathrm{CO})$ were determined. $\mathrm{CO}$ was measured by both thermodilution and Fick method. Simultaneously, all patients underwent direct assessment of LV filling pressures, followed by coronary angiography. Derived hemodynamic parameters were calculated according to standard formulae [18]. PH was diagnosed according to recent guidelines as $\mathrm{mPAP} \geq 25 \mathrm{mmHg}$ at rest [19]. PH was classified as moderate or severe when the mPAP was $\geq 30 \mathrm{mmHg}$. The transpulmonary pressure gradient (TPG) was defined as difference between mPAP and PAWP and the diastolic pressure gradient (DPG) as difference between diastolic PA pressure and PAWP.

\section{Cardiovascular magnetic resonance}

All CMR studies were performed on a 1.5- $\mathrm{T}$ scanner (Avanto, Siemens Medical Solutions, Erlangen, Germany) within 30 days of RHC (mean $14.8 \pm 9.0$ days). Studies consisted of functional and Late Gadolinium Enhancement (LGE) imaging, according to standard protocols [20]. For cine imaging, steady state free precision (SSFP) images were used (repetition time $3.2 \mathrm{~ms}$, echo time $1.2 \mathrm{~ms}$, flip angle $64^{\circ}$, voxel size $1.4 \times 1.4 \times 6 \mathrm{~mm}$, matrix $180 \times 256)$. For LGE imaging of the left ventricle, segmented inversion recovery sequences (repetition time: $700 \mathrm{~ms}$, echo time $1.22 \mathrm{~ms}$, flip angle $50^{\circ}$, voxel size $1.4 \times$ $1.4 \times .8 \mathrm{~mm}$, matrix $146 \times 256$ ) were acquired $10 \mathrm{~min}$ after injection of $0.1 \mathrm{mmol} / \mathrm{kg}$ gadolinium-DTPA (Gadovist'; Bayer Vital GmbH, Leverkusen, Germany).

PA and Ao diameters were measured from inner-edge to inner-edge in axial half-Fourier single-shot turbo spinecho (HASTE) black blood images (repetition time $810 \mathrm{~ms}$, echo time $43 \mathrm{~ms}$, flip angle $160^{\circ}$, voxel size $1.6 \times$ $1.6 \times 5 \mathrm{~mm}$, matrix $192 \times 256)$ at the level of the PA bifurcation (Fig. 1).

For SMT measurements, cine images were analyzed. The largest area and the longest medio-lateral diameter of the septal insertion part of SMT as identified in the 


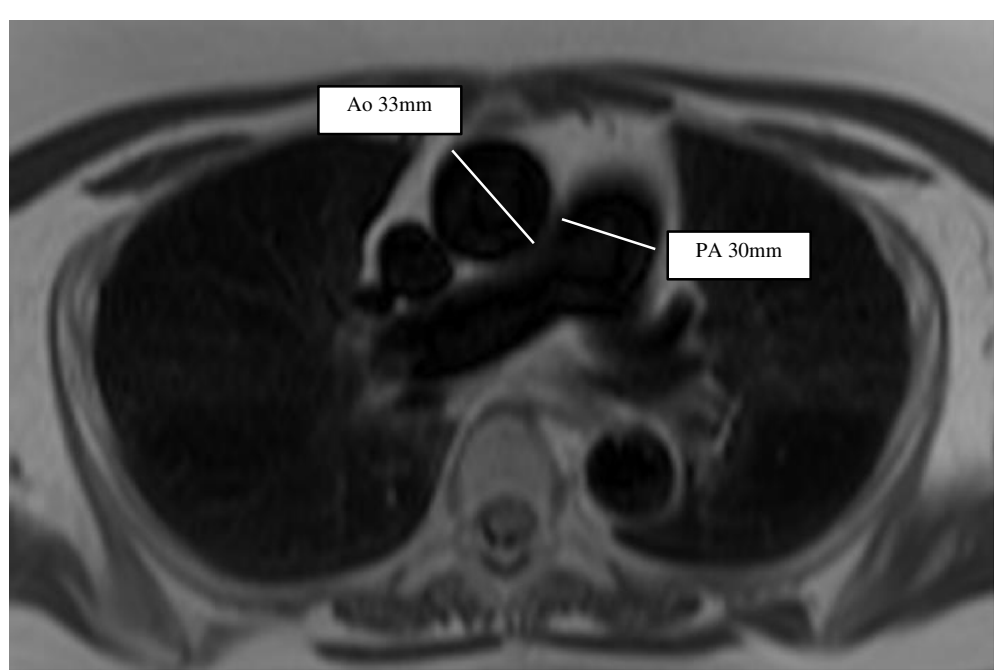

Fig. 1 Example for the measurement of pulmonary artery (PA) and ascending aorta (Ao) diameters in the axial black blood sequence in a patient with a mean pulmonary artery pressure of $33 \mathrm{mmHg}$

second or third basal slice of the short axis cine stack were measured (Fig. 2). The RVFW was measured in end-diastole in the same short axis image used for SMT quantification.

All CMR studies were read by two independent observers blinded to clinical data (GK, SP and JB).

\section{Outcome measures}

Patients were prospectively followed by ambulatory visits and telephone calls at 6-month intervals. The main outcome measure was a combined endpoint consisting of hospitalization for heart failure or death from cardiac causes. Endpoints were ascertained by follow-up visits and phone calls.

\section{Statistical analysis}

Categorical data are presented as total numbers or percent, and continuous variables as mean \pm standard deviation. Chi square test or Fisher's exact test were applied for categorical variables and the Wilcoxon two-sample test for continuous variables. Kaplan-Meier estimates were used to calculate cardiac event rates. Differences between Kaplan-Meier curves were analyzed using the log rank test.

Binary logistic regression analysis was used to identify variables associated with moderate/severe $\mathrm{PH}$ (mPAP $\geq 30 \mathrm{mmHg}$ ). In addition, a univariable Cox regression analysis was used to identify parameters (SMT diameter, SMT area, RVFW, PA size and PA:Ao ratio) associated

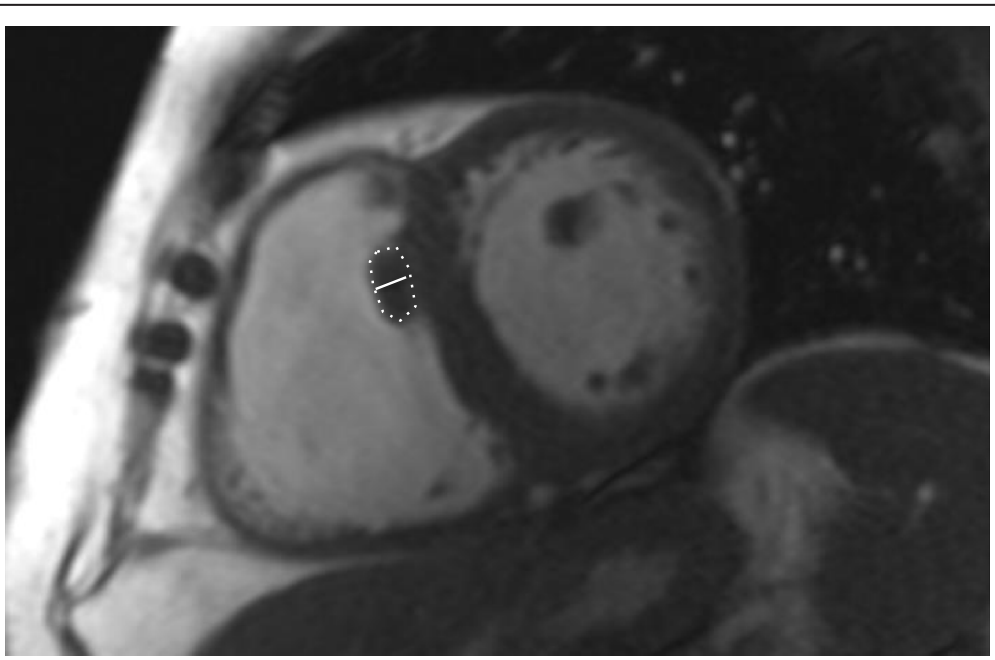

Fig. 2 Example for the measurement of the septomarginal trabeculation (SMT) diameter and area in an axial cine image in a patient with a mean pulmonary artery pressure of $33 \mathrm{mmHg}$ 
with event-free survival. In a second step these parameters were adjusted for potential cofounding clinical variables such as age, sex, atrial fibrillation, arterial hypertension, diabetes, chronic obstructive pulmonary disease, and coronary artery disease. All variables with a $p$-value $<0.05$ in the univariable model were entered into a multivariable model using a stepwise approach. Hazard and odds ratios for the PA:Ao ratio were reported as PA diameter divided by Ao diameter times 100. Receiver Operating Characteristic (ROC) curves were used to determine cut-off values for the detection of moderate/severe $\mathrm{PH}$. Interobserver variability was described by using Intra Class Correlation Coefficients (ICCs). Statistical analyses were performed with SPSS Statistics version 18 (IBM, Armonk, New York). Results were considered statistically significant if $p$-values were $<0.05$.

\section{Results}

Baseline characteristics

Between 2010 and 2013159 consecutive patients were enrolled. Of these, 48 had to be excluded from the study because CMR was not possible due to pacemakers, GFR $<30 \mathrm{~mL} / \mathrm{min} / 1.73 \mathrm{~m}^{2}$, or claustrophobia. Of the remaining 111 patients (32.4\% male, $70 \pm 9$ years old) who underwent CMR, $64 \%$ suffered from moderate or severe $\mathrm{PH}(\mathrm{mPAP} \geq 30 \mathrm{mmHg}$ by RHC). Clinical characteristics, hemodynamic measurements, and CMR parameters of registered patients are summarized in Table 1.

With respect to baseline clinical parameters, patients with moderate/severe $\mathrm{PH}$ more frequently presented with diabetes (50.0\% versus $23.1 \%, p=0.006)$, non-significant coronary artery disease/previous revascularization (27.1\% versus $5.1 \%, p=0.005)$; shorter 6 -min walk distances (301 $\pm 110 \mathrm{~m}$ versus $397 \pm 104 \mathrm{~m}, p<0.001)$ and had more dilated left atria (LA; $66.7 \pm 9.3 \mathrm{~mm}$ versus $62.9 \pm$ $8.7 \mathrm{~mm}, p=0.035$ for diameters and $33.5 \pm 10.2 \mathrm{~cm}^{2}$ versus $28.3 \pm 6.8 \mathrm{~cm}^{2}, p=0.005$ for areas).

Invasive assessment showed significant differences between patients with and without moderate/severe $\mathrm{PH}$ with respect to all measured variables except for $\mathrm{CO}$, which was similar in both groups $(5.2 \pm 1.2$ versus $5.2 \pm 1.5 \mathrm{l} / \mathrm{min}$, $p=0.829$.

60 (54\%) patients had a TPG >12 mmHg (formerly classified as "out-of-proportion PH") and 13 (12\%) had a DPG $\geq 7 \mathrm{mmHg}$, indicating combined pre- and postcapillary pulmonary hypertension [21].

Patients with a TPG $>12 \mathrm{mmHg}$ had a significantly higher PA:Ao ratio as compared to patients with a TPG $\leq 12 \mathrm{mmHg}(0.9 \pm 0.2$ versus $0.8 \pm 0.2, p=0.001)$ whereas PA:Ao ratios did not differ between patients with a DPG of $\geq$ and $<7 \mathrm{mmHg}(0.9 \pm 0.2$ versus $0.9 \pm 0.2, p=0.230)$. However, this lack in statistical significance may be due to the small proportion of patients with DPGs $\geq 7 \mathrm{mmHg}$ (12\% of the study cohort).
LGE was present in $31 \%(n=34)$ of our patients and involved $8.8 \pm 4.3 \%$ of left ventricular myocardial mass. In the majority $(n=22)$, LGE was present at the insertion point of the right ventricle; 8 patients had midmyocardial and 4 subendocardial enhancement. There were no significant differences in LGE pattern or degree between groups with and without moderate/severe $\mathrm{PH}$.

\section{Dimensions of the great arteries and indices of right ventricular hypertrophy for the prediction of moderate/ severe pulmonary hypertension}

Dimensions of the great arteries and indices of RV hypertrophy by CMR are displayed in Table 2. Patients with moderate/severe $\mathrm{PH}$ showed significantly larger PA diameters than the comparator $(30.9 \pm 5.1 \mathrm{~mm}$ versus $26 \pm 5.1 \mathrm{~mm}, p<0.001)$ and also had significantly higher PA:Ao ratios $(0.93 \pm 0.2$ versus $0.79 \pm 0.1, p<0.001)$. SMT diameters $(4.6 \pm 1.2 \mathrm{~mm}$ versus $3.8 \pm 1.2 \mathrm{~mm}, p=$ 0.008 ) were larger in patients with moderate/severe $\mathrm{PH}$, while areas did not differ between groups $(p=0.136)$. RVFW showed a wide range from 2.0 to $7.2 \mathrm{~mm}(4.2 \pm$ $1.1 \mathrm{~mm}$ ) and did also not differ between patients with and without moderate/severe $\mathrm{PH}(p=0.183)$. PA:Ao ratio, PA diameter and SMT diameter were significantly correlated with mPAP $(r=0.421, p<0.001 ; r=0.401, p<0.001 ; r=$ $0.267, p=0.005$; respectively). Fig. 3 displays the correlation of PA:Ao ratio with mPAP.

Table 3 shows the binary logistic regression model for predicting the presence of moderate/severe $\mathrm{PH}$. By univariable analysis, SMT diameter $(p=0.010)$, PA diameter $(p<0.001)$, and PA:Ao ratio $(p<0.001)$ were associated with moderate/severe $\mathrm{PH}$. However, by multivariable analysis only PA:Ao ratio remained independently associated with the presence of moderate/severe $\mathrm{PH}$.

By ROC analysis, a PA:Ao ratio of 0.83 was found to best detect mPAP $\geq 30 \mathrm{mmHg}$ with a sensitivity of $73.2 \%$ and a specificity of $67.5 \%$, and an area under the curve (AUC) of 0.759 (Fig. 4).

Measurements of PA:Ao ratio and PA diameter showed excellent inter-observer agreement with an Intra Class Correlation Coefficient (ICC) of 0.815 and 0.905 while ICCs for SMT diameters were only 0.563 , and for RVFW 0.581.

\section{Cardiac outcomes}

Patients were followed for $22.0 \pm 14.9$ months. During follow-up 40 cardiac events (34 hospitalizations for heart failure, 6 deaths from cardiovascular causes) occurred.

Table 4 shows the results of the multivariable Cox-regression model. By univariable analysis, SMT diameter $(p=0.023)$, RVFW $(p=0.034)$, PA diameter $(p=0.002)$, and PA:Ao ratio $(p=0.003)$ were significantly associated with cardiovascular events. After adjustment for age, sex, atrial fibrillation, diabetes, chronic 
Table 1 Baseline patient characteristics

\begin{tabular}{|c|c|c|c|c|}
\hline Variable & mPAP $<30 \mathrm{mmHg} 40$ patients (36 \%) & mPAP $\geq 30 \mathrm{mmHg} 71$ patients (64 \%) & All 111 patients & $p$ value \\
\hline \multicolumn{5}{|l|}{ Clinical parameters } \\
\hline Age [years] & $68.9 \pm 10.6$ & $70.6 \pm 8.4$ & $70.0 \pm 9.3$ & 0.348 \\
\hline Male sex [\%] & 32.5 & 32.4 & 32.4 & 0.991 \\
\hline $\mathrm{BSA}\left[\mathrm{m}^{2}\right]$ & $1.9 \pm 0.3$ & $1.9 \pm 0.2$ & $1.9 \pm 0.2$ & 0.367 \\
\hline AFib [\%] & 51.3 & 67.1 & 61.5 & 0.103 \\
\hline Diabetes [\%] & 23.1 & 50.0 & 40.4 & 0.006 \\
\hline $\mathrm{HbA1c}[\%]$ & $5.9 \pm 0.6$ & $6.3 \pm 1.0$ & $6.2 \pm 0.9$ & 0.028 \\
\hline Smoking [\%] & 33.3 & 43.3 & 39.6 & 0.312 \\
\hline $\mathrm{CAD}^{*}[\%]$ & 5.1 & 27.1 & 19.3 & 0.005 \\
\hline Hypertension [\%] & 94.9 & 100.0 & 98.2 & 0.056 \\
\hline BMI $\left[\mathrm{kg} / \mathrm{m}^{2}\right]$ & $29.3 \pm 6.3$ & $31.4 \pm 6.0$ & $30.7 \pm 6.1$ & 0.079 \\
\hline Heart rate [beats/min] & $72.2 \pm 14.8$ & $70.8 \pm 13.3$ & $71.4 \pm 13.8$ & 0.626 \\
\hline 6-MWD [m] & $396.7 \pm 104.3$ & $301.0 \pm 110.2$ & $334.4 \pm 117.0$ & $<0.001$ \\
\hline GFR $\left[\mathrm{mL} / \mathrm{min} / 1.73 \mathrm{~m}^{2}\right]$ & $64.9 \pm 22.7$ & $59.10 \pm 17.4$ & $61.0 \pm 19.5$ & 0.142 \\
\hline NT-proBNP [pg/ml] & $1363.8 \pm 2127.4$ & $2252.1 \pm 3746.1$ & $1934.8 \pm 3278.6$ & 0.171 \\
\hline NYHA & & & & 0.081 \\
\hline II [\%] & 41.0 & 23.2 & 29.6 & \\
\hline III [\%] & 56.4 & 66.7 & 63.0 & \\
\hline IV [\%] & 2.6 & 10.1 & 7.4 & \\
\hline \multicolumn{5}{|c|}{ Hemodynamic parameters } \\
\hline $\mathrm{mPAP}[\mathrm{mmHg}]$ & $23.9 \pm 3.6$ & $39.1 \pm 8.0$ & $33.7 \pm 10.0$ & $<0.001$ \\
\hline $\mathrm{sPAP}[\mathrm{mmHg}]$ & $36.7 \pm 6.1$ & $61.5 \pm 15.4$ & $52.6 \pm 17.5$ & $<0.001$ \\
\hline $\mathrm{dPAP}[\mathrm{mmHg}]$ & $15.9 \pm 4.0$ & $25.3 \pm 6.2$ & $21.9 \pm 7.1$ & $<0.001$ \\
\hline PAWP [mmHg] & $15.5 \pm 3.3$ & $22.1 \pm 4.3$ & $19.7 \pm 5.1$ & $<0.001$ \\
\hline LVEDP $[\mathrm{mmHg}]$ & $16.1 \pm 4.6$ & $22.4 \pm 6.0$ & $20.3 \pm 6.3$ & $<0.001$ \\
\hline PVR [dyne* sec/cm & $134.8 \pm 67.4$ & $276.9 \pm 125.1$ & $226.5 \pm 127.7$ & $<0.001$ \\
\hline $\mathrm{PAC}[\mathrm{ml} / \mathrm{mmHg}]$ & $3.9 \pm 2.1$ & $2.3 \pm 0.9$ & $2.9 \pm 1.7$ & $<0.001$ \\
\hline $\mathrm{CO}[\mathrm{l} / \mathrm{min}]$ & $5.3 \pm 1.5$ & $5.2 \pm 1.2$ & $5.2 \pm 1.3$ & 0.829 \\
\hline \multicolumn{5}{|c|}{ Cardiovascular magnetic resonance imaging parameters } \\
\hline LV EDD [mm] & $46.5 \pm 4.7$ & $47.8 \pm 6.3$ & $47.3 \pm 5.8$ & 0.259 \\
\hline LV EDV [ml] & $117.7 \pm 33.5$ & $133.5 \pm 52.6$ & $127.9 \pm 47.1$ & 0.057 \\
\hline LV EDVi $\left[\mathrm{ml} / \mathrm{m}^{2}\right]$ & $62.4 \pm 18.4$ & $68.3 \pm 23.7$ & $66.2 \pm 22.0$ & 0.160 \\
\hline LV EF [\%] & $62.7 \pm 10.6$ & $63.3 \pm 11.9$ & $63.1 \pm 11.4$ & 0.813 \\
\hline LV mass $[\mathrm{g}]$ & $108.4 \pm 37.6$ & $117.5 \pm 36.1$ & $113.0 \pm 36.8$ & 0.250 \\
\hline IVS [mm] & $11.5 \pm 2.6$ & $11.6 \pm 2.2$ & $11.5 \pm 2.3$ & 0.924 \\
\hline LA diameter $[\mathrm{mm}]$ & $62.9 \pm 8.7$ & $66.7 \pm 9.3$ & $65.3 \pm 9.2$ & 0.035 \\
\hline LA area $\left[\mathrm{cm}^{2}\right]$ & $28.3 \pm 6.8$ & $33.5 \pm 10.2$ & $31.6 \pm 9.4$ & 0.005 \\
\hline RV EDD [mm] & $38.9 \pm 5.9$ & $40.8 \pm 8.1$ & $40.1 \pm 7.4$ & 0.193 \\
\hline RV EDV [ml] & $143.4 \pm 39.6$ & $160.0 \pm 67.1$ & $151.3 \pm 51.7$ & 0.109 \\
\hline RV EDVi $\left[\mathrm{ml} / \mathrm{m}^{2}\right]$ & $76.1 \pm 20.7$ & $80.5 \pm 29.2$ & $78.9 \pm 26.4$ & 0.453 \\
\hline RV EF [\%] & $50.9 \pm 9.1$ & $52.5 \pm 11.3$ & $51.9 \pm 10.6$ & 0.452 \\
\hline RA diameter [mm] & $63.5 \pm 8.7$ & $66.7 \pm 8.9$ & $65.5 \pm 9.0$ & 0.072 \\
\hline $\mathrm{RA}$ area $\left[\mathrm{cm}^{2}\right]$ & $28.3 \pm 8.9$ & $30.5 \pm 8.8$ & $29.7 \pm 8.9$ & 0.221 \\
\hline LGE present [\%] & 25.0 & 34.8 & 31.2 & 0.288 \\
\hline
\end{tabular}


Table 1 Baseline patient characteristics (Continued)

\begin{tabular}{|c|c|c|c|c|}
\hline Amount of LGE LE** $^{* *}[\mathrm{c}$ & $6.8 \pm 2.7$ & $9.6 \pm 4.7$ & $8.8 \pm 4.3$ & 0.093 \\
\hline Subendocardial [\%] & 0 & 5.8 & 3.7 & $0.294^{* *}$ \\
\hline Midmyocardial [\%] & 2.5 & 10.1 & 7.3 & $0.254^{* *}$ \\
\hline RV insertion point [\%] & 22.5 & 18.8 & 20.2 & 0.646 \\
\hline \multicolumn{5}{|c|}{$\begin{array}{l}B S A \text { indicates body surface area, } A F i b \text { atrial fibrillation, } C A D \text { coronary artery disease, } B M I \text { body mass index, } 6-M W D \text { six } \\
\text { filtration rate, NYHA New York Heart Association functional class, } m P A P \text { mean pulmonary artery pressure, sPAP systolic } \\
\text { pulmonary artery pressure, PAWP pulmonary artery wedge pressure, } L V E D P \text { left ventricular end-diastolic pressure, } P V R \text { p } \\
\text { artery compliance, } C O \text { cardiac output, } L V \text { left ventricle, } E D D \text { end-diastolic diameter, EDV end-diastolic volume, } i \text { indexed to b } \\
\text { IVS interventricular septum thickness, } L A \text { left atrium, } R V \text { right ventricle, } R A \text { right atrium, } L G E \text { Late Gadolinium Enhancement } \\
{ }^{*} \text { non-significant coronary artery disease or prior revascularization } \\
{ }^{* *} \text {-values derive from Fisher's exact test }\end{array}$} \\
\hline
\end{tabular}

obstructive pulmonary disease and coronary artery disease, PA diameter $(p=0.003)$, and PA:Ao ratio $(p=0.002)$ remained significant predictors of outcome. However, after multivariable adjustment, only PA:Ao ratio remained independently associated with event-free survival.

By Kaplan Meier analysis (Fig. 5), event-free survival was significantly worse in patients with a PA:Ao ratio $\geq 0.83$ (log rank, $p=0.004$ ).

\section{Discussion}

The present study demonstrates that the PA:Ao ratio can be used as a simple and straight-forward indicator of $\mathrm{PH}$ associated with HFpEF. Moreover, the PA:Ao ratio is of prognostic value with respect to cardiac events.

In patients suffering from HFpEF right heart variables are of particular interest as they play an important role in terms of prognosis [5, 22-27]. Echocardiography is the first, and often only imaging modality used in HFpEF patients due to its wide availability. However, the RV is not easy to examine by echo and considerable inter- and intraobserver variability exists [9]. Furthermore, estimation of pulmonary hemodynamics may be inaccurate [8]. The definite diagnosis of $\mathrm{PH}$ demands invasive $\mathrm{RHC}$ to directly measure PA and wedge pressure, as well as trans-pulmonary and diastolic pulmonary pressure gradients. Although considered reasonably safe in experienced centers, RHC still carries a risk of morbidity and mortality [28]. Given the close link between pressures in the pulmonary circulation and RV dimensions and performance, several right heart imaging parameters have been shown to indicate the presence of $\mathrm{PH}[10,13,16$, 26, 29-35]. CMR has been established as the goldstandard technique for the assessment of the right heart $[10,36]$. It has the advantage that in addition to accurate and reproducible evaluation of heart size, function, and myocardial tissue characterization, CMR allows the assessment of the dimensions of the great arteries.

Indices of right ventricular hypertrophy for the prediction of pulmonary hypertension

RV mass and RV mass to LV mass ratio (VMI) have been shown to correlate reasonably well with mPAP in previous studies $[33,34]$, but the estimation of RV mass from CMR images is time-consuming and limited by significant inter- and intra-observer variability [16, 32]. Furthermore, it requires dedicated software for off-line analysis.

RVFW thickness is much more easy to assess than RV mass or VMI. In a historical series this parameter appeared useful as surrogate marker of $\mathrm{PH}$ [30] and was also shown to be of prognostic value [31]. However, due to the hypertrabeculated nature of the RV considerable rater-dependent variation exists for this measurement, which significantly limits its usefulness [30]. In the present series, RVFW measurements showed weak reproducibility and did not differentiate between patients with and without moderate/severe $\mathrm{PH}$.

Table 2 Dimensions of the great arteries and indices of right ventricular hypertrophy for the prediction of moderate/severe pulmonary hypertension

\begin{tabular}{|c|c|c|c|c|}
\hline Variable & mPAP $<30$ mmHg 40 patients (36 \%) & mPAP $\geq 30 \mathrm{mmHg} 71$ patients (64 \%) & All 111 patients & $p$-value \\
\hline PA diameter $[\mathrm{mm}]$ & $26.3 \pm 5.1$ & $30.9 \pm 5.1$ & $29.2 \pm 5.5$ & $<0.001$ \\
\hline Ao diameter $[\mathrm{mm}]$ & $33.6 \pm 4.3$ & $33.4 \pm 4.1$ & $33.5 \pm 4.1$ & 0.856 \\
\hline PA:Ao ratio & $0.79 \pm 0.1$ & $0.93 \pm 0.2$ & $0.89 \pm 0.2$ & $<0.001$ \\
\hline SMT diameter $[\mathrm{mm}]$ & $3.8 \pm 1.2$ & $4.6 \pm 1.2$ & $4.3 \pm 1.4$ & 0.008 \\
\hline SMT area $\left[\mathrm{mm}^{2}\right]$ & $0.96 \pm 0.3$ & $1.11 \pm 0.5$ & $1.06 \pm 0.4$ & 0.136 \\
\hline RVFW [mm] & $4.0 \pm 1.2$ & $4.3 \pm 1.1$ & $4.2 \pm 1.2$ & 0.183 \\
\hline
\end{tabular}

PA indicates pulmonary artery, Ao ascending aorta, RVFW right ventricular free wall, SMT septomarginal trabeculation 


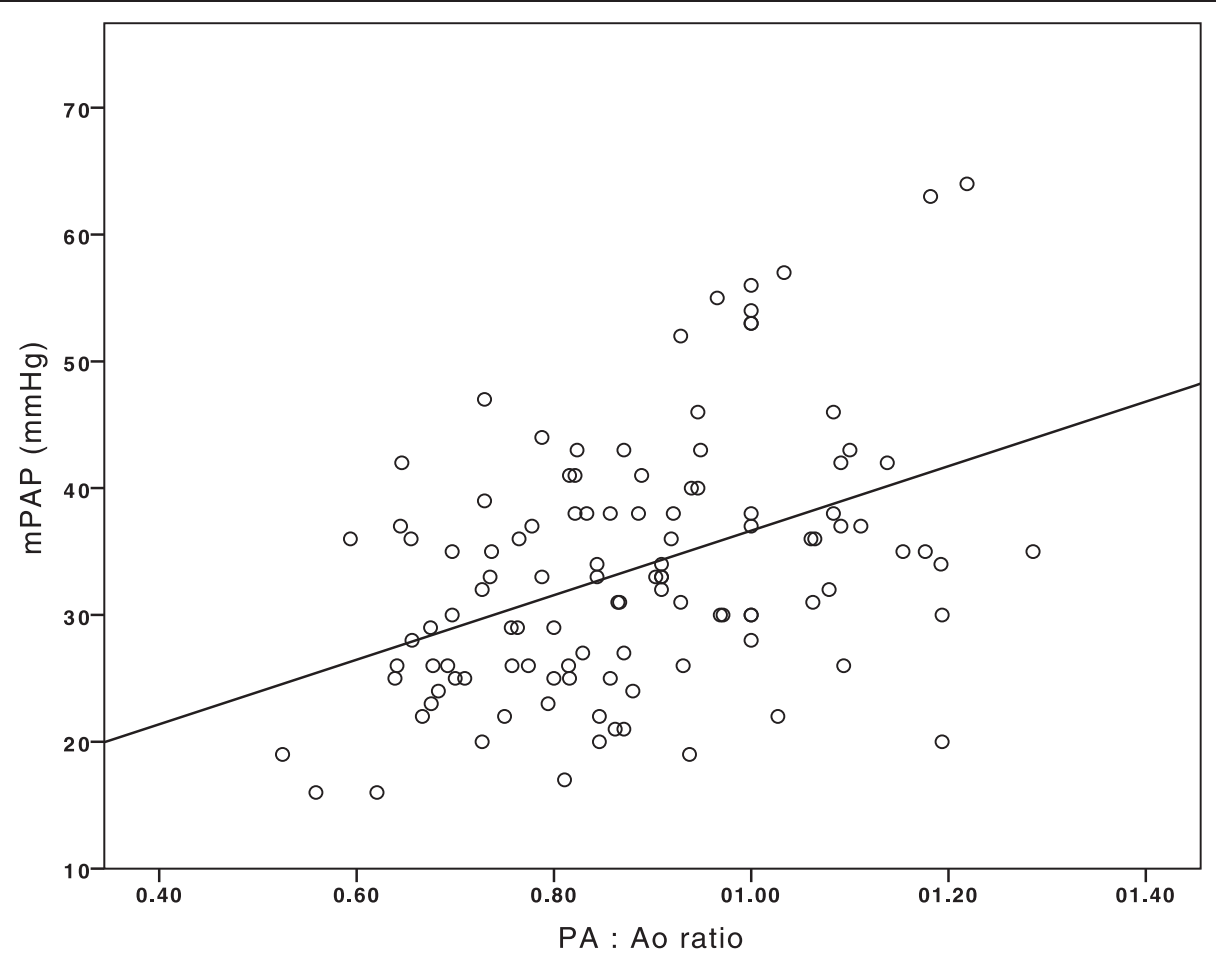

Fig. 3 Scatter plot showing the correlation between pulmonary artery to ascending aorta (PA:Ao) ratio and mean pulmonary artery pressure (mPAP; $r=0.421, p<0.001)$

The SMT is a prominent muscle band that originates from the basal ventricular septum and runs apical-laterally towards the RV free wall [37]. A recent retrospective CMR study on a small number of patients $(n=49)$ found that SMT mass could be a useful marker for the identification of $\mathrm{PH}$ [16]. However, in the present study SMT was hard to delineate in many patients, leading to significant rater-

Table 3 Binary logistic regression model for the presence of moderate/severe pulmonary hypertension (mean pulmonary artery pressure $\geq 30 \mathrm{mmHg}$ )

\begin{tabular}{llllll}
\hline & Regr.Coeff. & $p$-value & OR & $95 \%$ Cl for OR \\
\hline Univariable & & & & & \\
SMT area & 0.853 & 0.090 & 2.346 & 0.876 & 6.282 \\
SMT diameter & 0.404 & $\mathbf{0 . 0 1 0}$ & 1.498 & 1.103 & 2.034 \\
RVFW & 0.236 & 0.200 & 1.267 & 0.882 & 1.818 \\
PA diameter & 0.175 & $<0.001$ & 1.191 & 1.091 & 1.301 \\
Ao diamter & -0.008 & 0.875 & .992 & 0.903 & 1.090 \\
PA:Ao x 100 & 0.066 & $<0.001$ & 1.068 & 1.035 & 1.103 \\
RVEDV & 0.005 & 0.239 & 1.005 & 0.997 & 1.013 \\
RVEF & 0.017 & 0.377 & 1.018 & 0.979 & 1.058 \\
Multivariable & & & & & \\
PA:Ao x 100 & 0.066 & $<\mathbf{0 . 0 0 1}$ & 1.068 & 1.035 & 1.103 \\
\hline
\end{tabular}

SMT indicates septomarginal trabeculation, RVFW right ventricular free wall, $P A$ pulmonary artery, Ao ascending aorta, RVEDV right ventricular end-diastolic volume, RVEF right ventricular ejection fraction dependent variation. Its diagnostic power for the identification of patients with moderate/severe $\mathrm{PH}$ was clearly inferior as compared to PA:Ao ratio and PA diameter.

\section{Dimensions of the great arteries for the prediction of pulmonary hypertension}

In contrast to the aforementioned parameters (RV myocardial mass, RVFW, SMT), the size of PA and Ao are easy to measure. Such measurements do not require dedicated software or advanced expertise. In the present study PA:Ao ratio was identified as the most potent predictor for the presence of moderate/severe PH. It correlated better with mPAP than PA diameter or indices of RV hypertrophy and showed excellent reproducibility. In addition, PA:Ao ratio was independently associated with event-free survival. From a clinical perspective the differentiation between isolated post-capillary $\mathrm{PH}$ and combined forms is crucial, in particular with regard to prognosis [21] and therapeutic consequences. The TPG and DPG have been introduced to quantify pulmonary vascular disease occurring as a consequence of PAWP elevation. In the present study, a greater PA:Ao ratio was predictive for TPG >12 mmHg.

Several previous studies have evaluated the usefulness of the PA:Ao ratio for the detection of $\mathrm{PH}$ [11-15]. However, only Chan and coworkers report data from a population with $\mathrm{PH}$ due to left heart disease [11]. In that 


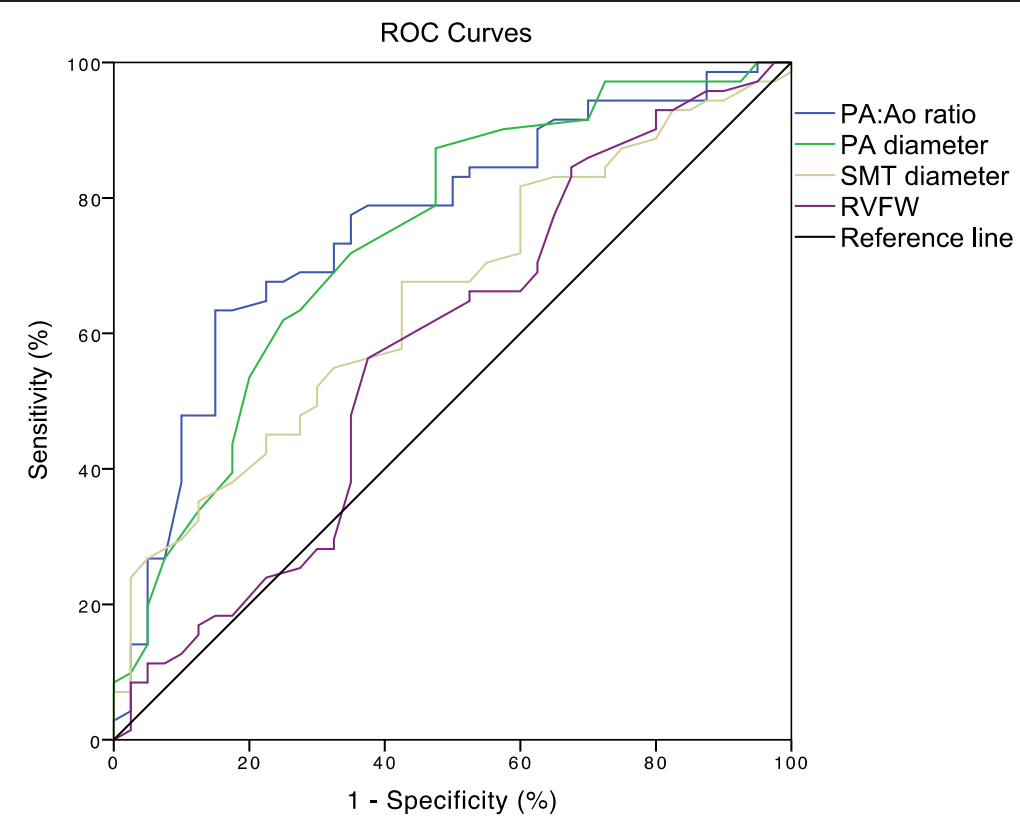

Fig. 4 Receiver Operating Characteristics (ROC) curves of pulmonary artery to ascending aorta (PA:Ao) ratio, pulmonary artery (PA) diameter, septomarginal trabeculation (SMT) diameter and right ventricular free wall thickness (RVFW) with Areas Under the Curve of 0.759, $0.741,0.652$ and 0.576 , respectively

Table 4 Univariable, univariable adjusted, and multivariable adjusted Cox-regression model for cardiovascular events

\begin{tabular}{|c|c|c|c|c|c|}
\hline & Regr.Coeff. & $p$-value & $H R$ & \multicolumn{2}{|c|}{$95.0 \% \mathrm{Cl}$} \\
\hline \multicolumn{6}{|l|}{ Univariable } \\
\hline SMT area & 0.663 & 0.069 & 1.941 & 0.950 & 3.963 \\
\hline SMT diameter & 0.253 & 0.023 & 1.287 & 1.035 & 1.602 \\
\hline RVFW & 0.282 & 0.034 & 1.326 & 1.022 & 1.720 \\
\hline PA diameter & 0.093 & 0.002 & 1.097 & 1.035 & 1.163 \\
\hline PA:Ao x 100 & 0.027 & 0.003 & 1.027 & 1.010 & 1.046 \\
\hline RVEDV & 0.003 & 0.365 & 1.003 & 0.997 & 1.009 \\
\hline RVEF & -0.016 & 0.340 & 0.985 & 0.954 & 1.017 \\
\hline \multicolumn{6}{|l|}{ Adjusted* } \\
\hline SMT area & 0.500 & 0.180 & 1.650 & 0.793 & 3.430 \\
\hline SMT diameter & 0.233 & 0.060 & 1.262 & 0.990 & 1.608 \\
\hline RVFW & 0.357 & 0.052 & 1.428 & 0.997 & 2.047 \\
\hline PA diameter & 0.126 & 0.003 & 1.135 & 1.044 & 1.233 \\
\hline PA:Ao x 100 & 0.034 & 0.002 & 1.035 & 1.013 & 1.057 \\
\hline RVEDV & 0.004 & 0.298 & 1.004 & 0.997 & 1.011 \\
\hline RVEF & -0.032 & 0.095 & 0.968 & 0.933 & 1.006 \\
\hline \multicolumn{6}{|c|}{ Multivariable, adjusted* } \\
\hline PA:Ao x 100 & 0.031 & 0.003 & 1.031 & 1.010 & 1.053 \\
\hline
\end{tabular}

SMT indicates septomarginal trabeculation, RVFW right ventricular free wall, $P A$ pulmonary artery, Ao ascending aorta, RVEDV right ventricular end-diastolic volume

*Adjusted for age, sex, atrial fibrillation, diabetes, chronic obstructive pulmonary disease and coronary artery disease retrospective analysis of 101 patients the authors defined a PA:Ao ratio cut-off of 0.84 for the detection of $\mathrm{PH}$ (mPAP $>25 \mathrm{mmHg}$ ), which is in line with our results.

Other studies have used a PA:Ao ratio cut-off of $>1.0$ for the suspicion of $\mathrm{PH}$. These included 60 patients with chronic obstructive pulmonary disease [13], 81 patients with connective tissue disease [15], 50 patients with mainly pulmonary disease [14], and a mixed population of 175 patients [12]. The differences with respect to the PA:Ao cutoff for the diagnosis of $\mathrm{PH}$ may be due to 1) retrospective design of all aforementioned studies, 2) heterogeneity of cut-offs for the diagnosis of $\mathrm{PH}$ (mPAP $>20 \mathrm{mmHg}$ [14] versus $\mathrm{mPAP}>25 \mathrm{mmHg}[11-13,15]), 3$ ) heterogeneity of diagnoses underlying $\mathrm{PH}$, and 4) small patient numbers [13-15]. Taken together, it remains to be determined whether the results of the present study are applicable in patient populations other than HFpEF.

\section{Limitations}

Presented data have been collected in a single center setting. Therefore, a center-specific bias cannot be excluded. However, the major advantages of limiting data collection to a single center are 1 . inclusion of a homogenous patient population, 2. adherence to a constant clinical routine, 3 . constant quality of echocardiographic and CMR work-up and 4. constant follow-up.

PA and Ao diameters were acquired from axial HASTE black blood images and were not planned exactly in line with the vessel orientation. This may have led to some over- or underestimation of the vessel size. 


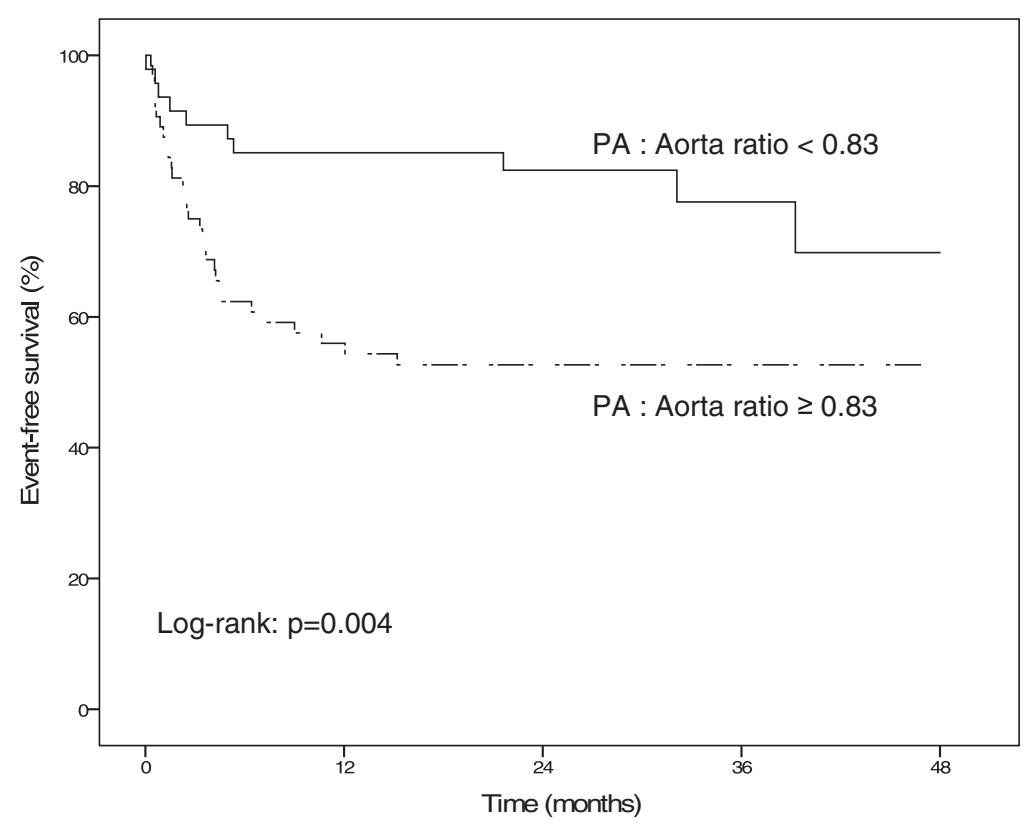

\begin{tabular}{|c|c|c|c|c|}
\hline Year & 1 & 2 & 3 & 4 \\
\hline \multicolumn{5}{|c|}{ PA:Ao ratio $<0.83(n=47)$} \\
\hline Number at risk & 47 & 40 & 26 & 15 \\
\hline Event $(n)$ & 7 & 1 & 1 & 1 \\
\hline Survival (\%) & 85 & 83 & 79 & 69 \\
\hline \multicolumn{5}{|c|}{ PA : Ao ratio $\geq 0.83(n=64)$} \\
\hline Number at risk & 64 & 35 & 21 & 7 \\
\hline Event (n) & 28 & 2 & 0 & 0 \\
\hline Survival (\%) & 56 & 52 & 52 & 52 \\
\hline
\end{tabular}

Fig. 5 Kaplan-Meier analysis according to pulmonary artery to ascending aorta ratio. Patients with a pulmonary artery to ascending aorta (PA:Ao) ratio $<0.83$ showed a significantly better event-free survival than patients with a PA:Ao ratio $\geq 0.83$

The majority of patients included in this study were suffering from $\mathrm{PH}$ ( $83.8 \%$ had $\mathrm{mPAP} \geq 25 \mathrm{mmHg})$. Sixty four percent had at least moderate $\mathrm{PH}$ (mPAP $\geq 30 \mathrm{mmHg}$ ). Due to the small number of patients in the group without $\mathrm{PH}$, patients were dichotomized in groups with mPAPs above and below $30 \mathrm{mmHg}$, defined as groups with no/mild $\mathrm{PH}$ versus moderate/severe $\mathrm{PH}$. However, previous work shows that a PA:Ao ratio of 0.84 also discriminates between patients with mPAPs above and below $25 \mathrm{mmHg}$ [11]. In addition to morphological indicators of right heart pressure overload, recent data indicate the feasibility of CMR to estimate pulmonary hemodynamics, such as MPAP and pulmonary vascular resistance by using numerical models [35]. Such estimates, however, include various assumptions. Furthermore, they require extensive experience of the investigator. Thus, the accuracy and widespread applicability of these measurements still need to be proven. Furthermore, we did not analyse RV mass, which might have augmented the diagnostic performance of the PA:Ao ratio.
However, in the present work we aimed to identify parameters that are easy to measure and do not require dedicated software or extensive training.

\section{Conclusions}

PA:Ao ratio is an easily assessable, straight-forward marker of $\mathrm{PH}$ in HFpEF patients. Moreover, it is strongly associated with outcome. Other CMR derived indices of RV pressure overload such as RVFW or SMT thickness are less accurate and reproducible.

\section{Competing interests}

The authors declare that they have no competing interests.

\section{Authors' contributions}

GK, JB and SP were responsible for data acquisition. AAK and JM were responsible for data analysis and interpretation. JM and DB were responsible for the study design. AAK, GK and JM drafted the manuscript. SA, BAM, CZ, $A B, A D, F D, J B, S P$ and $D B$ critically revised the manuscript. All authors read and approved the final manuscript. 


\section{Funding sources}

This study received support from the Austrian Society of Cardiology (to JM), the Österreichischer Herzfonds (to JM) and the Austrian fellowship grants KLI 246 (to DB), and KLI 245 (to JM).

\section{Author details}

${ }^{1}$ Department of Cardiology, Medical University of Vienna, Waehringer Guertel 18-20, 1090 Vienna, Austria. ${ }^{2}$ Acibadem Maslak Hospital, Istanbul, Turkey. ${ }^{3}$ Otto Wagner Hospital, Vienna, Austria.

\section{Received: 19 March 2015 Accepted: 16 August 2015}

Published online: 30 August 2015

\section{References}

1. Guazzi M. Pulmonary hypertension in heart failure preserved ejection fraction: Prevalence, pathophysiology, and clinical perspectives. Circ Heart Fail. 2014;7:367-77.

2. Lee DS, Gona P, Vasan RS, Larson MG, Benjamin EJ, Wang TJ, et al. Relation of disease pathogenesis and risk factors to heart failure with preserved or reduced ejection fraction: Insights from the framingham heart study of the national heart, lung, and blood institute. Circulation. 2009;119:3070-7.

3. Vachiery JL, Adir Y, Barbera JA, Champion H, Coghlan JG, Cottin V, et al. Pulmonary hypertension due to left heart diseases. J Am Coll Cardiol. 2013;62:D100-8.

4. Lam CS, Roger VL, Rodeheffer RJ, Borlaug BA, Enders FT, Redfield MM. Pulmonary hypertension in heart failure with preserved ejection fraction: A community-based study. J Am Coll Cardiol. 2009;53:1119-26.

5. Zotter-Tufaro C, Mascherbauer J, Duca F, Koell B, Aschauer S, Kammerlander $A A$, et al. Prognostic significance and determinants of the six-minute walk test in patients with heart failure and preserved ejection fraction. JACC Heart Fail. 2014; [accepted for publication, \#JHF082514-0348-TR]

6. Mascherbauer J, Marzluf BA, Tufaro C, Pfaffenberger S, Graf A, Wexberg P, et al. Cardiac magnetic resonance postcontrast t1 time is associated with outcome in patients with heart failure and preserved ejection fraction. Circ Cardiovasc Imaging. 2013:6:1056-65.

7. Perez VA, Haddad F, Zamanian RT. Diagnosis and management of pulmonary hypertension associated with left ventricular diastolic dysfunction. Pulm Circ. 2012;2:163-9.

8. Fisher MR, Forfia PR, Chamera E, Housten-Harris T, Champion HC, Girgis RE, et al. Accuracy of doppler echocardiography in the hemodynamic assessment of pulmonary hypertension. Am J Respir Crit Care Med. 2009;179:615-21.

9. Rudski LG, Lai WW, Afilalo J, Hua L, Handschumacher MD, Chandrasekaran K, et al. Guidelines for the echocardiographic assessment of the right heart in adults: a report from the american society of echocardiography endorsed by the european association of echocardiography, a registered branch of the european society of cardiology, and the canadian society of echocardiography. J Am Soc Echocardiogr. 2010;23:685-713. quiz 786-688.

10. McLure LE, Peacock AJ. Cardiac magnetic resonance imaging for the assessment of the heart and pulmonary circulation in pulmonary hypertension. Eur Respir J. 2009;33:1454-66.

11. Chan AL, Juarez MM, Shelton DK, MacDonald T, Li CS, Lin TC, et al. Novel computed tomographic chest metrics to detect pulmonary hypertension. BMC Med Imaging. 2011;11:7.

12. Corson N, Armato 3rd SG, Labby ZE, Straus C, Starkey A, Gomberg-Maitland M. Ct-based pulmonary artery measurements for the assessment of pulmonary hypertension. Acad Radiol. 2014;21:523-30.

13. Iyer AS, Wells JM, Vishin S, Bhatt SP, Wille KM, Dransfield MT. Ct scanmeasured pulmonary artery to aorta ratio and echocardiography for detecting pulmonary hypertension in severe copd. Chest. 2014;145:824-32

14. $\mathrm{Ng}$ CS, Wells AU, Padley SP. A ct sign of chronic pulmonary arterial hypertension: The ratio of main pulmonary artery to aortic diameter J Thorac Imaging. 1999;14:270-8.

15. Rajaram S, Swift AJ, Capener D, Elliot CA, Condliffe R, Davies C, et al Comparison of the diagnostic utility of cardiac magnetic resonance imaging, computed tomography, and echocardiography in assessment of suspected pulmonary arterial hypertension in patients with connective tissue disease. J Rheumatol. 2012;39:1265-74

16. Vogel-Claussen J, Shehata ML, Lossnitzer D, Skrok J, Singh S, Boyce D, et al, Increased right ventricular septomarginal trabeculation mass is a novel marker for pulmonary hypertension: Comparison with ventricular mass index and right ventricular mass. Invest Radiol. 2011;46:567-75.

17. Paulus WJ, Tschope C, Sanderson JE, Rusconi C, Flachskampf FA Rademakers FE, et al. How to diagnose diastolic heart failure: a consensus statement on the diagnosis of heart failure with normal left ventricular ejection fraction by the heart failure and echocardiography associations of the european society of cardiology. Eur Heart J. 2007;28:2539-50.

18. Baim D. Grossman's cardiac catheterization, angiography, and intervention Philadelphia, PA: Lippincott Williams \& Wilkins; 2006.

19. Galie N, Hoeper MM, Humbert M, Torbicki A, Vachiery JL, Barbera JA, et al. Guidelines for the diagnosis and treatment of pulmonary hypertension: the task force for the diagnosis and treatment of pulmonary hypertension of the european society of cardiology (esc) and the european respiratory society (ers), endorsed by the international society of heart and lung transplantation (ishlt). Eur Heart J. 2009:30:2493-537.

20. Kramer CM, Barkhausen J, Flamm SD, Kim RJ, Nagel E. Standardized cardiovascular magnetic resonance (cmr) protocols 2013 update. J Cardiovasc Magn Reson. 2013;15:91

21. Zotter-Tufaro C, Duca F, Kammerlander A, Koell B, Aschauer S, Dalos D, et al Diastolic pressure gradient predicts outcome in patients with heart failure and preserved ejection fraction. J Am Coll Cardiol. 2015; [accepted for publication. JACC030215-0720RR]

22. Morris DA, Gailani M, Vaz Perez A, Blaschke F, Dietz R, Haverkamp W, et al. Right ventricular myocardial systolic and diastolic dysfunction in heart failure with normal left ventricular ejection fraction. J Am Soc Echocardiogr. 2011;24:886-97.

23. Mohammed SF, Hussain I, Abou Ezzeddine OF, Takahama H, Kwon SH, Forfia $\mathrm{P}$, et al. Right ventricular function in heart failure with preserved ejection fraction: A community based study. Circulation. 2014;130:2310.

24. Burke MA, Katz DH, Beussink L, Selvaraj S, Gupta DK, Fox J, et al. Prognostic importance of pathophysiologic markers in patients with heart failure and preserved ejection fraction. Circ Heart Fail. 2014;7:288-99.

25. Melenovsky V, Hwang SJ, Lin G, Redfield MM, Borlaug BA. Right heart dysfunction in heart failure with preserved ejection fraction. Eur Heart J. 2014;35:3452-62

26. Aschauer S, Zotter-Tufaro C, Kammerlander AA, Pfaffenberger S, Marzluf BA, Bonderman $\mathrm{D}$, et al. The right heart in hfpef, insights from a cardiac magnetic resonance study. Eur Heart J. 2014;35 (Abstract Supplement):532.

27. Goliasch G, Tufaro C, Aschauer S, Kammerlander A, Pfaffenberger S, Mascherbauer J, et al. Outcome in heart failure with preserved ejection fraction strongly depends on right ventricular performance. Eur Heart J. 2014;35(Abstract Supplement):1036.

28. Hoeper MM, Lee SH, Voswinckel R, Palazzini M, Jais X, Marinelli A, et al. Complications of right heart catheterization procedures in patients with pulmonary hypertension in experienced centers. J Am Coll Cardiol. 2006:48:2546-52.

29. Linguraru MG, Pura JA, Gladwin MT, Koroulakis Al, Minniti C, Machado RF, et al. Computed tomography correlates with cardiopulmonary hemodynamics in pulmonary hypertension in adults with sickle cell disease. Pulm Circ. 2014:4:319-29.

30. Bouchard A, Higgins CB, Byrd 3rd BF, Amparo EG, Osaki L, Axelrod R. Magnetic resonance imaging in pulmonary arterial hypertension. Am J Cardiol. 1985;56:938-42.

31. Ghio S, Pazzano AS, Klersy C, Scelsi L, Raineri C, Camporotondo R, et al. Clinical and prognostic relevance of echocardiographic evaluation of right ventricular geometry in patients with idiopathic pulmonary arterial hypertension. Am J Cardiol. 2011;107:628-32.

32. Pattynama PM, Lamb HJ, Van der Velde EA, Van der Geest RJ, Van der Wal EE, De Roos A. Reproducibility of mri-derived measurements of right ventricular volumes and myocardial mass. Magn Reson Imaging 1995;13:53-63.

33. Roeleveld RJ, Marcus JT, Boonstra A, Postmus PE, Marques KM, Bronzwaer $J G$, et al. A comparison of noninvasive mri-based methods of estimating pulmonary artery pressure in pulmonary hypertension. J Magn Reson Imaging. 2005;22:67-72.

34. Saba TS, Foster J, Cockburn M, Cowan M, Peacock AJ. Ventricular mass index using magnetic resonance imaging accurately estimates pulmonary artery pressure. Eur Respir J. 2002;20:1519-24.

35. Swift AJ, Rajaram S, Hurdman J, Hill C, Davies C, Sproson TW, et al. Noninvasive estimation of pa pressure, flow, and resistance with $\mathrm{cmr}$ 
imaging: Derivation and prospective validation study from the aspire registry. JACC Cardiovasc Imaging. 2013;6:1036-47.

36. Grapsa J, O'Regan DP, Pavlopoulos H, Durighel G, Dawson D,

Nihoyannopoulos P. Right ventricular remodelling in pulmonary arterial hypertension with three-dimensional echocardiography: Comparison with cardiac magnetic resonance imaging. Eur J Echocardiogr. 2010;11:64-73.

37. Kosinski A, Kozlowski D, Nowinski J, Lewicka E, Dabrowska-Kugacka A, Raczak $G$, et al. Morphogenetic aspects of the septomarginal trabecula in the human heart. Arch Med Sci. 2010;6:733-43.

\section{Submit your next manuscript to BioMed Central} and take full advantage of:

- Convenient online submission

- Thorough peer review

- No space constraints or color figure charges

- Immediate publication on acceptance

- Inclusion in PubMed, CAS, Scopus and Google Scholar

- Research which is freely available for redistribution 